\title{
Pregeometric modelling of the spacetime phenomenology
}

\author{
Reginald T. Cahill and Christopher M. Klinger \\ Department of Physics, Flinders University * \\ GPO Box 2100, Adelaide 5001, Australia
}

May 1996

\begin{abstract}
At present we have only the very successful but phenomenological Einstein geometrical modelling of the spacetime phenomenon. This geometrical model provides a 'container' for other theories, in particular the quantum field theories. Here we report progress in developing a Heraclitean Quantum System. This is a particular pregeometric theory for space and time in which no classical or geometric structures are assumed, but rather the emergence of such phenomena is sought.
\end{abstract}

Ta panta rei - all is flux

Heraclitus of Ephesus, sixth century BC

*E-mail: Reg.Cahill@flinders.edu.au, Chris.Klinger@flinders.edu.au 
1. Introduction At present we have no theory of the phenomena of time and space. Rather we have a very successful phenomenology given to us by Einstein. We regard Einstein's model as a phenomenology for the simple reason that in setting up this model one makes very explicit assumptions about time and space. For example one of the key Einstein assumptions was to assume, in addition to the very phenomenon of time, that time is local, which contrasted sharply with Newton's assumption of a global time. We take the defining indicator of a theory to be the property that it predicts phenomena, but does not have those phenomena explicitly or even covertly built into its axioms. Well known examples of successful theories include the atomic theory: it predicted the existence and properties of atoms, molecules, etc, but contained only electrons and nuclei in the axioms. A second example is that of nuclear physics: Quantum Chromdynamics (QCD) begins with quarks and gluons and predicts the phenomena of hadrons, nuclei etc. In this example there was a long period of phenomenological modelling in which hadrons were described by effective actions, or equivalently Hamiltonians, involving hadronic fields. The structure of these effective actions was obtained by appealing to various symmetries that appeared to be manifested in the hadronic data. These examples illustrate the idea of emergent phenomena.

A feature of the Einstein model is that it is a geometrical model, and is a generalisation of the geometrical modelling by Galileo and Newton. These models build upon the different Ancient Greek models of Pythagoras, Parmenides and Democritus. This modelling is so effective and persuasive that there is a tendency to confuse the phenomena of time and space with the geometrical modelling. For example the modelling of time, whether local or global, by the real number line is often implicitly assumed to be an actual property of the phenomenon of time.

The present standard model of physics, while successful, has a very strange three stage structure. First one constructs a classical geometrical spacetime structure. Second, various classical fields are attached to this geometrical structure, and finally, in the third stage one quantises the matter fields. As an afterthought, one might even attempt to derive the classical behaviour of large quantum systems by means of some classicalisation argument. What one sees in this structuring is an incomplete separation of the historical development of the subject from a proper theoretical structure. In a mature theory one would expect to see the classical features as emergent properties of some abstract quantum system which itself does not contain classical structures. We call such systems Heraclitean Quantum Systems (HQS) after Heraclitus of Ephesus (540-480 BC) who appears to have anticipated such ideas by some 2500 years. He argued that common sense is mistaken in thinking that the world consists of stable things; rather the world is in a state of flux. The appearance of 'things' depends upon this flux for their continuity and identity. What needs to be explained, Heraclitus argued, is not change, but the appearance of stability. We suggest that the success of the standard model in its present three stage form is a clear indication of some extremely robust mean-field type phenomena arising within a 
HQS. Rather than a co-evolving unified system, our three-stage modelling amounts to first evolving space with at most only a macroscopic input from the matter energy density, and then in a second computational sweep we evolve the quantum matter. While certainly a very effective approximation it nevertheless makes it impossible for correlations in the quantum processing of the spatial phenomenon to influence the quantum matter processing. Indeed, while it is this approximation which makes possible local field theories, it also renders these same theories with short distance problems.

In recent years much effort has been put into attempts to quantise gravity. To us this seems a suspect procedure for discovering deeper theories. Quantisation is not a fundamental physical process, rather it is a guessing procedure that has been invoked somewhat fruitfully in the last 70 years. To give a recent counter example we note that the quark-gluon quantum system was not obtained by quantising the classical hadronic phenomenological field theory of the 1960s. Rather the next level down from hadrons was obtained by some inspired guessing. The problems that then arose were the demonstration that hadronic laws, in the form of an effective action description, could be extracted from the quark-gluon system, and also the experimental study of hadronic systems to reveal signatures of the quark-gluon subsystem.

Of considerable current interest is the process of classicalisation. In this one attempts to deduce a special emergent behaviour of large quantum systems. In such systems classical behaviour is a weird and poorly understood phenomenon. The obvious fact that we modelled classical behaviour first does not deny the fact that it is a secondary effect. Basic quantum mechanics is still bedevilled by the metaphysical fix-ups that were invoked in the early days of quantum theory when studying the transition from a simple to a complex large scale quantum system that occurs during the measurement process. Fortunately classicality is now seen as a physical process requiring detailed dynamical analysis. Quantisation is not de-classicalisation. By quantising some classical emergent phenomenon we do not recover the deeper quantum system that produced that classicality.

The need to construct a non-geometric theory to explain the time and space phenomena has been strongly argued by Wheeler [1], under the name of pregeometry. Gibbs [2] has recently compiled a literature survey of such attempts. They include: cellular automata, lattice field theories, quantum metric spaces, causal nets, poset models, simplicial quantum gravity, fractals, topological quantum field theory, field theory on a complex cell, spin networks, twistor theory, signal spaces, non-commutative geometry and event-symmetric space-time. Isham [3] has recently discussed the possibility that spacetime is indeed a phenomenological construct, and not fundamental.

Here we give a brief outline of some of the insights that have been obtained for a particular Heraclitean Quantum System, which is a pregeometric type model with no classical structures assumed in its axioms. In many pregeometric models residual classical and phenomenological structures are retained. Our HQS is an abstract Grassmannian algebraic system, which is based upon some of the insights gained from the derivation [4] of the 
emergent hadronic phenomena for the quark-gluon system. The nature of this analysis is briefly discussed in section 2 , and highlights the idea of action sequencing induced by dynamically determined changes of functional integration variables. In section 3 a particular HQS is presented together with some insight into how spacetime might arise.

2 Action Sequencing QCD provides us with a fine example of the emergence of complex effective theories. Some parts exhibit an induced geometrical form, while overall we see the idea of action sequencing that is an integral part of the derivation of emergent phenomena, and also the importance of condensate effects. These results have been achieved using the Functional Integral Calculus (FIC) [4] which most powerfully takes advantage of the functional integral formulation of quantum field theories.

QCD essentially involves the functional integral in (1) for the vacuum persistence amplitude in the presence of sources $J$ (which are not shown on the RHS). At low energies or long wavelengths we only observe hadronic degrees of freedom, and not the quark and gluon fields. In this respect we expect QCD to be archetypal: in HQS we do not expect to observe the fundamental defining algebraic elements. Their usefulness will rest solely upon their role in successfully predicting a large amount of higher level observable phenomena. The derivation of the low energy form of QCD, namely the hadronic form, is outlined in (11)-(值). As expected this derivation is not exact. A useful step is that of approximating QCD by the Global Colour Model (GCM) 四.

$$
\begin{aligned}
<0 \mid 0>_{J} & =\int \mathcal{D} \bar{q} \mathcal{D} q \mathcal{D} A \exp \left(-S_{Q C D}[A, \bar{q}, q]\right) \\
& \approx \int \mathcal{D} \bar{q} \mathcal{D} q \mathcal{D} A \exp \left(-S_{G C M}[A, \bar{q}, q]\right) \quad(\text { Global Colour Model }) \\
& \left.=\int \mathcal{D} \mathcal{B} \mathcal{D} \mathcal{D} \mathcal{D} \mathcal{D}^{\star} \exp \left(-S_{b l}\left[\mathcal{B}, \mathcal{D}, \mathcal{D}^{\star}\right]\right) \quad \text { (bilocal fields }\right) \\
& =\int \mathcal{D} \pi \ldots \mathcal{D} \bar{N} \mathcal{D} N \ldots \exp \left(-S_{\text {had }}[\pi, \ldots, \bar{N}, N, . .]\right) \quad \text { (local fields) }
\end{aligned}
$$

The derived hadronic action that finally emerges from this action sequencing, to low order in fields and derivatives, has the form

$$
\begin{array}{r}
S_{\text {had }}[\pi, \ldots, \bar{N}, N, . .]= \\
\int d^{4} x \operatorname{tr}\left\{\bar{N}\left(\gamma . \partial+m_{N}+\Delta m_{N}-m_{N} \sqrt{ } 2 i \gamma_{5} \pi^{a} \mathcal{T}^{a}+. .\right) N\right\}+ \\
+\int d^{4} x\left(\frac{f_{\pi}^{2}}{4} \operatorname{tr}\left(\partial_{\mu} U \partial_{\mu} U^{\dagger}\right)+\kappa_{1} \operatorname{tr}\left(\partial^{2} U \partial^{2} U^{\dagger}\right)+\frac{\rho}{2} \operatorname{tr}\left(\left[\mathbf{1}-\frac{U+U^{\dagger}}{2}\right] \mathcal{M}\right)+\right. \\
+\kappa_{2} \operatorname{tr}\left(\left[\partial_{\mu} U \partial_{\mu} U^{\dagger}\right]^{2}\right)+\kappa_{3} \operatorname{tr}\left(\partial_{\mu} U \partial_{\nu} U^{\dagger} \partial_{\mu} U \partial_{\nu} U^{\dagger}\right)+ \\
+\frac{f_{\omega}^{2}}{2}\left[-\omega_{\mu} \square \omega_{\mu}+\left(\partial_{\mu} \omega_{\mu}\right)^{2}+m_{\omega}^{2} \omega_{\mu}^{2}\right]+\frac{f_{\rho}^{2}}{2}\left[-\rho_{\mu} \square \rho_{\mu}+\left(\partial_{\mu} \rho_{\mu}\right)^{2}+m_{\rho}^{2} \rho_{\mu}^{2}\right]+
\end{array}
$$




$$
\begin{array}{r}
-f_{\rho} f_{\pi}^{2} g_{\rho \pi \pi} \rho_{\mu} . \pi \times \partial_{\mu} \pi-i f_{\omega} f_{\pi}^{3} \epsilon_{\mu \nu \sigma \tau} \omega_{\mu} \partial_{\nu} \pi . \partial_{\sigma} \pi \times \partial_{\tau} \pi+ \\
-i f_{\omega} f_{\rho} f_{\pi} G_{\omega \rho \pi} \epsilon_{\mu \nu \sigma \tau} \omega_{\mu} \partial_{\nu} \rho_{\sigma} . \partial_{\tau} \pi+ \\
\left.+\frac{i}{80 \pi^{2}} \epsilon_{\mu \nu \sigma \tau} \operatorname{tr}\left(\pi . F \partial_{\mu} \pi . F \partial_{\nu} \pi . F \partial_{\sigma} \pi . F \partial_{\tau} \pi . F\right)+\ldots . .\right)
\end{array}
$$

This shows that the emergent hadronic phenomena are very rich and complex; that is why the nucleus is so much more complicated than atoms.

We see in the above the powerful notion of action sequencing

$$
S_{Q C D}[A, \bar{q}, q] \rightarrow S_{G C M}[A, \bar{q}, q] \rightarrow S_{b l}\left[\mathcal{B}, \mathcal{D}, \mathcal{D}^{\star}\right] \rightarrow S_{h a d}[\pi, \ldots, \bar{N}, N, . .]
$$

Each change of functional integration field variables, and these are mandated by the dynamics, generates a new effective action for those field variables. It is only the final hadronic variables and their induced effective action that allows us to relate QCD to the experimental data. Even the hadronic form in (舟) requires further evaluation to produce the physical hadrons, since (四) involves the so-called core or constituent states. The final hadronic functional integration dresses each of these core states with a cloud of other hadrons, mainly low mass mesons.

A key intermediate step is the determination of the minimum of the action in (3)

$$
\frac{\delta S_{b l}\left[\mathcal{B}, \mathcal{D}, \mathcal{D}^{\star}\right]}{\delta \mathcal{B}}=0, \ldots
$$

which has a solution with $\mathcal{B} \neq 0$ and gives the $\bar{q} q$ condensate effect. This simply means that the induced effective action has a non-trivial minimum away from the perturbative $\mathcal{B}=0$ point. Similar effects occur in superconductivity. This condensate effect is one of the most important dynamical effects in QCD and goes a long way in explaining the nature of hadrons. In particular it generates a running mass for the constituent quarks, and leads to the constituent quark mass of some $300 \mathrm{MeV}$. A recent account is given in [5]. The structure of the condensate and the consequent structure of the hadrons is determined by the gluon correlations. At the end of the calculation of the derivative expansion we, in effect, suppress any explicit mention of the internal structure of the hadrons, resulting in local couplings of local fields - the emergent hadronic phenomenon.

Hadrons may be viewed as deviations in the structure of the condensate. The lowest mass hadrons correspond to those deviations in the flattest directions of the effective action for the bilocal fields, in (3). These correspond to the pions. If the quark current masses are zero then these are directions in which the action is strictly flat, and the resulting massless mesons are known as Nambu - Goldstone (NG) bosons. These massless modes are represented in (5) by the matrix $U(x)=\exp \left(i \sqrt{ } 2 \pi^{a}(x) F^{a}\right)$ where the $\left\{F^{a}\right\}$ are the generators of the $S U\left(N_{f}\right)$ flavour symmetry group. The NG boson fields $\pi(x)$ form homogeneous Riemann coordinates for this vacuum manifold, which has the form of a coset space. The internal structure of the pions is intimately related to the structure of the condensate. Thus the long range part of the nuclear force is determined by the near 
degeneracy of the condensate equations (7). See [6] for a recent analysis of the pion sector of the GCM. We end this section by giving an insight into the nature of the condensate deviations. If $\overline{\mathcal{B}}_{0}(x, y)$ is a particular solution of $(\overline{7})$, possibly having degenerate solutions, then the idea of a condensate deviation is given by

$$
\mathcal{B}(x, y)=\overline{\mathcal{B}}_{0}(x, y)+\sum_{a} \phi_{a}\left(\frac{x+y}{2}\right) \Gamma^{a}(x-y)
$$

in which we expand the $x-y$ dependence of $\mathcal{B}(x, y)$ into a complete set $\Gamma^{a}(w)$, with the $\phi_{a}(z)$ as expansion coefficients. We may change the variables of integration from the $\mathcal{B}(x, y)$ to the $\phi_{a}$. In the GCM the $\Gamma^{a}(w)$ are chosen in order to diagonalize the 2nd order terms arising when the bilocal effective in (3) is expanded about $\overline{\mathcal{B}}_{0}(x, y)$. This essentially leads to the $\Gamma^{a}(w)$ being solutions of Bethe-Salpeter equations, and describing the internal structure of $\bar{q} q$ core states. The fields $\phi_{a}(z)$ describe the 'centre-of-mass' motion of these mesonic bound states. Fields of this type occur in (4). This expansion procedure leads to a bosonisation of QCD. A detailed formal derivation and generalization to introduce diquarks and baryons is given in [四, leading to the hadronisation of QCD. Recent results and discussion are given in [5].

3. Heraclitean Quantum Systems A HQS has no classical structures or concepts built into the axioms. We will consider a model with only abstract algebraic elements. This algebra is taken to be a Grassmann algebraic system. Such algebras are used very effectively to model the fermionic sector of the standard model, though in these applications they are mutli-component local 'algebraic fields' attached to some spacetime manifold. Here we ask whether such an abstract system can induce our very successful spacetime phenomenology together with the quantum matter modelling of our present standard model. Can we adapt the bosonisation techniques developed in the GCM to discover any classical features that such a system might possess? The Grassmann algebra retains, as an assumed intrinsic property, an abstract form of the Pauli Exclusion Principle, which is realised via the anti-commuting property of the algebra. The algebra is a set of $2 \mathrm{~N}$ elements, with eventually $N \rightarrow \infty$.

$$
\left\{M_{i}, i=1,2, \ldots, 2 N\right\}=\left\{\bar{m}_{i}, m_{i}, i=1,2, \ldots, N\right\}
$$

and, by definition, mutually anticommuting

$$
M_{i} M_{j}=-M_{j} M_{i} \quad \text { so that } \quad M_{i} M_{i}=0
$$

The subscript explicitly labels these simple elements: there are no hidden indices. The distinction between $\bar{m}_{i}$ and $m_{i}$ only arises when some form for the 'action' $S_{H Q S}[\bar{m}, m]$ is specified. We name $\bar{m}, m$ monads after Leibniz [0]. An abstract notion of 'correlation' is defined by

$$
G_{j \ldots}^{i, . .}=\mathcal{G}\left[\bar{m}_{i} m_{j} \ldots e^{-S_{H Q S}[\bar{m}, m]}\right]
$$


which involves Grassmann 'integration'. $\mathcal{G}[\ldots]$ is a purely algebraic process [8]. Such extremely primitive 'correlations' are not expected to have in themselves any phenomenological significance. The phenomena we are looking for must be manifested with respect to the many complex co-phenomena that a HQS must produce in order to be a viable theory. That is, we must develope an 'internal view' of the emergent phenomena. Unlike our present quantum modelling we cannot take an 'external view'. The basic 'processing' of the HQS is based on the assumption that the Grassmannian modelling of the fermionic sector of the standard model, with its quantum correlations being determined by a Grassmannian 'integration', is a residual property of an underlying HQS. Indeed we have seen in the GCM that this Grassmannian modelling of the quarks re-emerges, at a higher level, as a Grassmannian description of the baryons.

A more general 'correlation' is

$$
G[F]=\mathcal{G}\left[F[\bar{m}, m] e^{-S_{H Q S}[\bar{m}, m]}\right]
$$

where $F$ is some function of all the elements. To define the integration process we expand the 'integrand' as a polynomial

$$
F[\bar{m}, m] e^{-S_{H Q S}[\bar{m}, m]}=1+\sum c_{i} m_{i}+\ldots+c_{L} \bar{m}_{1} \bar{m}_{2} \ldots \bar{m}_{N} m_{1} m_{2} \ldots m_{N}
$$

The sum of the terms of highest order has been written in some standard order. Then by definition,

$$
\mathcal{G}\left[F[\bar{m}, m] e^{-S_{H Q S}[\bar{m}, m]}\right]=c_{L}
$$

A particular Grassmann integration that can be explicitly performed is [8]

$$
\mathcal{G}\left[e^{-\sum M_{i} A_{i j} M_{j}}\right]=\operatorname{Pf}(2 A)
$$

where $A$ is an antisymmetric matrix, and where the $\operatorname{Pfaffian} \operatorname{Pf}(A)$ is the square root of the determinant of an antisymmetric matrix $A$, in the sense that $\operatorname{Pf}(A)^{2}=\operatorname{det} A$.

Extending the Grassmann algebra to include 'sources' $\bar{l}_{i}, l_{i}$, a generating functional is introduced

$$
Z[\bar{l}, l]=\mathcal{G}\left[e^{-S_{H Q S}[\bar{m}, m]-\bar{l} m-\bar{m} l}\right]
$$

Let us consider the particular HQS defined by the quartic action

$$
S_{H Q S}[\bar{m}, m]=-\frac{1}{2} \bar{m} \cdot m \bar{m} \cdot m=\sum_{i>j} \bar{m}_{i} m_{j} \bar{m}_{j} m_{i}
$$

No notion of locality is permissible, so all elements are in 'interaction'. The apparent dominance of local interactions must be emergent. The action has a large invariance group: $m \rightarrow U m, \bar{m} \rightarrow \bar{m} U^{-1}$. 
Consider a bosonisation along the line of the GCM bosonisation in QCD. We can put $Z$ in the form

$$
Z[\bar{l}, l]=\mathcal{G}\left[\int \mathcal{D} B e^{-S_{B m m}[B, \bar{m}, m]-\bar{l} m-\bar{m} l}\right]
$$

where

$$
S_{B m m}[B, \bar{m}, m]=\frac{1}{2} \sum_{i, j} B_{i j} B_{i j}-\sum_{i, j} B_{i j}\left(\bar{m}_{i} m_{j}-\bar{m}_{j} m_{i}\right)
$$

with $B_{i j}=-B_{j i}$ and real. This is easily checked on doing the gaussian $B$-integrations. We may now explicitly perform the $\mathcal{G}$ process giving

$$
Z[\bar{l}, l]=\int \mathcal{D} B e^{-\sum_{i>j} B_{i j}^{2}+\operatorname{Tr} \operatorname{Ln}(B)+\bar{l} B^{-1} l}
$$

The Grassmann algebraic aspects are now contained in TrLn and the $\bar{l}, l$ algebra. The algebraic $\mathcal{G}$ process has now been given a representation involving the sum over all possible $B$ configurations. This is the Heraclitean 'flux', and is an axiomatic aspect of the HQS. What is sought is the emergence of stability and 'things'. Or as Wheeler calls it "it from bit".

The induced action is

$$
S_{C}[B]=\sum_{i>j} B_{i j}^{2}-\operatorname{Tr} \operatorname{Ln}(B)
$$

Here ' $B$ ' is the analogue of bilocal fields in QCD. The advantage of the bosonisation is that it is more amenable to our well honed analytical techniques. As well our present day modelling has indicated the extraordinary success of mean field or smoothing approximations. We expect these to be accessible via the bosonisation. However note that the bosonisation does not preclude the emergence of complex fermionic components, as again illustrated by the GCM. The bosonisation technique now proceedes with an analysis of the minimum of the induced action. This identifies the most significant part of the $B$ integrations.

$$
\delta S_{C}[B] / \delta B=0
$$

gives $B=-B^{-1}$ - the 'condensate' equation, with solutions $\bar{B}$, analogous to the gap equation in superconductivity, and to the condensate equation in QCD. The general solution is $\bar{B}=R \bar{B}_{0} R^{-1}$ where $R$ is an arbitrary real orthogonal matrix and $\bar{B}_{0}$ is the block diagonal matrix

$$
\bar{B}_{0}=\left(\begin{array}{rrrrr}
0 & +1 & 0 & 0 & \ldots \\
-1 & 0 & 0 & 0 & \ldots \\
0 & 0 & +1 & 0 & \ldots \\
0 & -1 & 0 & 0 & \ldots
\end{array}\right)
$$


Hence the condensate is highly degenerate. The $R$ transformation 'switches' monad pairings. The degeneracy of the condensate dominates the $B$-fluctuations. We now search for signs of an emergent spacetime phenomenon, quantum fields, etc.

Consider the 'nihilo $\rightarrow$ nihilo amplitude'

$$
<\mathcal{N} \mid \mathcal{N}>=Z[0]=\int \mathcal{D} B e^{-S_{C}[B]}
$$

and the deviation from $\bar{B}_{0}$, in which the $\Gamma^{a}$ must be a complete set.

$$
B_{i j}=\bar{B}_{0 i j}+\sum_{a} \phi_{a} \Gamma_{i j}^{a}
$$

Our first choice for the $\Gamma_{i j}^{a}$ is the following: Set ' $a$ ' to be a serial index $a \equiv(I J)$, and with $\Gamma_{i j}^{a}=-\Gamma_{j i}^{a}=+1$ if $I=i$ and $J=j$, otherwise $\Gamma_{i j}^{a}=0$. These $\Gamma_{i j}^{a}$ form a complete set for the expansion, with expansion coefficients $\phi_{a}$. Then, changing variables of integration (the Jacobian is a constant and can be ignored),

$$
<\mathcal{N} \mid \mathcal{N}>=\int \mathcal{D} \phi e^{-S_{C}\left[\bar{B}_{0}+\phi . \Gamma\right]}
$$

This choice is essentially equivalent to the defining $B_{i j}$ integrations. As usual with degenerate condensates we make the superselection assumption that we can work in the neighbourhood of one condensate point, say $\bar{B}_{0}$, and expand $S_{C}$ in powers of $\phi_{a}$

$$
S_{C}\left[\bar{B}_{0}+\phi \cdot \Gamma\right]=S_{C}[0]+\sum_{a b} \phi_{a} \phi_{b} K_{a b}+\sum_{a b c} \phi_{a} \phi_{b} \phi_{c} K_{a b c}+. .
$$

where there is no linear term because of the condensate equation (22). These variables of integration affect only small numbers of $\bar{m}-m$ pairings. They are too primitive to be able to reveal any complex emergent behaviour. Nevertheless we can partly analyse them by choosing new variables of integration by diagonalising the quadratic term in (27), giving

$$
<\mathcal{N} \mid \mathcal{N}>=\int \mathcal{D} \Phi e^{-S_{C}[0]-\sum_{a} \Phi_{a} \Phi_{a} \lambda_{a}-\sum_{a b c} \Phi_{a} \Phi_{b} \Phi_{c} K_{a b c}^{\prime}+\ldots}
$$

This change of variables is equivalent to a new choice for the $\Gamma_{i j}^{a}$. Approximately one half of the eigenvalues $\lambda_{a}$ have value zero: these correspond to the 'massless' NG modes, i.e. deviations in the tangent plane to the condensate manifold. The remaining $\lambda_{a}$ are all non-zero and equal: these 'massive' modes correspond to deviations perpendicular to the condensate manifold. In QCD the analogue of the $\Phi_{a}$ modes are $\bar{q} q$ meson core-state modes, and the diagonalisation procedure is there the Bethe-Salpeter equation. Because of the peculiarities of QCD the hadrons contain either two constituent quarks (mesons) or three constituent quarks (baryons) together with secondary mesonic dressings of these core states. However in this HQS we are interested in multi-monad modes, within which we hope to find evidence of classical structures. For this purpose the above two possible choices of integration variables are not helpful. 
We now consider yet a third choice of integration variables. In (25) consider a new set of $\Gamma^{a}: \Gamma_{i j}^{a}=+1$ with probability $\frac{p}{2}$ or -1 with probability $\frac{p}{2}$, and $=0$ otherwise, i.e. with probability $q=1-p$. In some sense each such $\Gamma^{a}$ corresponds to some random multi-monad excitation of the condensate. We need this set to be complete. With the extreme choice $p=0$ only one trivial $\Gamma^{a}$ is formed. Similarly, if $p=1$ we form only $\Gamma^{\prime} \mathrm{s}$ with all off-diagonal entries being +1 or -1 . However if $p<<1$ then the $\Gamma^{a}$ have sparse non-zero entries, and approximate a complete set. Hence changing to these variables in (26), and using $G=\left\{G_{a}\right\}$ as the new variables of integration,

$$
<\mathcal{N} \mid \mathcal{N}>=\int \mathcal{D} G e^{-S_{C}\left[\bar{B}_{0}+G \cdot \Gamma\right]}
$$

This new set of multi-monad $\Gamma^{a}$ has a very interesting interpretation. To each such $\Gamma_{i j}^{a}$ matrix we can associate a random graph: consider the indices $i$ or $j$ as labelling the 'points' or 'nodes' of a graph, in which two points $i$ and $j$ are linked if $\left|\Gamma_{i j}^{a}\right|=1$. Such a graph is in general composed of disconnected pieces. Although we have no a priori background geometry we can nevertheless define one measure of distance between points within a connected piece by counting the minimum number of links connecting the points. Nagels 9] has considered the probability distribution of such distances in connected random graphs. Let $D_{k}=1,2,3, .$. be the number of points a distance $k=0,1,2,3, .$. from a particular arbitrary point, called the origin. So $D_{0}=1$ (by definition), $D_{1}$ is the number of adjacent points, etc. For $p<<1$ the shape of a connected random graph, as defined by the (relative) probability distribution of distances, is given by

$$
P\left[D_{k}\right]=\prod_{i=1}^{L} \frac{\left(D_{i-1}\right)^{D_{i}}}{D_{i} !}
$$

where $L$ is the maximum distance of any point from the origin. Further

$$
\sum_{k=0}^{L} D_{k}=N_{c}
$$

where $N_{c}$ is the number of points in the connected random graph. The most probable distribution, i.e. the most probable connected graph, from maximizing $P\left[D_{k}\right]$ subject to the constraint (31) [9] is

$$
D_{k} \sim \frac{L^{2} \ln L}{2 \pi^{2}}\left[\sin ^{2}\left(\frac{\pi k}{L}\right)-\frac{1}{3}\left(\frac{\pi}{L}\right) \sin \left(\frac{2 \pi k}{L}\right) \cdot \ln \sin \left(\frac{\pi k}{L}\right)\right]
$$

The remarkable property of the most probable distribution in (32) is that the resulting emergent structure closely resembles a three-dimensional closed space of positive curvature, for we obtain from (31) that

$$
N_{c} \sim\left(\frac{\ln L}{4 \pi^{2}}\right) L^{3}
$$


We also see the leading $\sin ^{2}$ term in (32) characteristic of the hypersphere $S^{3}$.

The partitioning of the random graphs into connected pieces is matched by a corresponding partitioning of each $\Gamma^{a}$, so that for the integration variables $G_{a}$ each subscript labels, in the most probable case, some three-dimensional kind of closed space, with internal structure specified by a $\Gamma^{a}$. After expanding the exponent in (29) in powers of $G_{a}$ and computing the trace summations, we are left with an induced effective action for the $G_{a}$

$$
<\mathcal{N} \mid \mathcal{N}>=\int \mathcal{D} G e^{-S[G]}
$$

corresponding to a quantum 'field' theory of interacting 3-spaces. This clearly has similarities with some programs that are currently being pursued in quantising general relativity. However it is not clear that our 3-spaces are necessarily to be directly identified with the spatial section of a 'universe', for one might expect to see some further condensation processes for these 'core' 3 -space states leading to a fractal or foamy spatial structure. From the point of view of quantising gravity Wheeler first pointed out that quantum fluctuations in the metric would give spacetime a foam-like structure. However in attempted quantisations of gravity the manifold point of view is maintained with considerable mathematical difficulty. Our HQS is not so constrained and a whole range of possible short distance behaviour is possible. Various short distance manifold descriptions have been studied by Hawking [10], Coleman [11] and others.

However the outstanding problem is to show that the HQS can induce a time phenomenon: can we demonstrate a natural classical sequencing - the basic phenomenon of time? To this end we introduce a complete set of functions $\left\{f_{\alpha}(G)\right\}$ for which

$$
\delta\left(G^{2}-G^{1}\right)=\sum_{\alpha} f_{\alpha}^{*}\left(G^{2}\right) f_{\alpha}\left(G^{1}\right)
$$

where now the superscript on $G^{i}$ labels different copies of $\left\{G_{a}\right\}$. Then

$$
\begin{aligned}
<\mathcal{N} \mid \mathcal{N}> & =\int \mathcal{D} G^{2} \int \mathcal{D} G^{1} e^{-\frac{1}{2} S\left[G^{2}\right]} \delta\left(G^{1}-G^{2}\right) e^{-\frac{1}{2} S\left[G^{1}\right]} \\
& =\sum_{\alpha} \int \mathcal{D} G^{2} 1 . e^{-S^{(2)}\left[G^{2}\right]} f_{\alpha}^{*}\left(G^{2}\right) \int \mathcal{D} G^{1} f_{\alpha}\left(G^{1}\right) e^{-S^{(2)}\left[G^{2}\right]} .1
\end{aligned}
$$

where we define, in general, $S^{(n)}[G]=\frac{1}{n} S[G]$. Think of

$$
C_{\alpha}^{(2)}=\int \mathcal{D} G^{1} f_{\alpha}\left(G^{1}\right) e^{-S^{(2)}\left[G^{1}\right]} \cdot 1
$$

as a transition amplitude, where the ' 1 ' represents the beginning of the 'universe', i.e. with all $G$ equally likely. Continue inserting complete sets

$$
C_{\alpha}^{(2)}=\sum_{\beta} \int \mathcal{D} G^{1} f_{\alpha}\left(G^{1}\right) e^{-S^{(4)}\left[G^{1}\right]} f_{\beta}^{*}\left(G^{1}\right) \int \mathcal{D} G^{2} f_{\beta}\left(G^{2}\right) e^{-S^{(4)}\left[G^{2}\right]} .1
$$




$$
\begin{aligned}
& C_{\alpha}^{(2)}=\sum_{\beta} A_{\alpha \beta}^{(4)} C_{\beta}^{(4)} \\
& A_{\alpha \beta}^{(n)}=\int \mathcal{D} G f_{\alpha}(G) e^{-S^{(n)}[G]} f_{\beta}^{*}(G)
\end{aligned}
$$

More insertions give

$$
C_{\alpha}^{(2)}=\sum_{\beta \gamma \ldots} A_{\alpha \beta}^{(n)} A_{\beta \gamma}^{(n)} \ldots C^{(n)}
$$

which has the form of an all-inclusive quantum-like multiple sequencing. But the time phenomenon is about restricted or classical sequencing, with only some residual quantum phenomena. A possible macroscopic unique sequencing or history is a partition of $\langle\mathcal{N} \mid \mathcal{N}\rangle$ such that different histories, by definition, have negligible interference. They are decoherent. They are classical. Hence we must look for a particular choice of complete set $\left\{f_{\alpha}(G)\right\}$ for which some of the members generate decoherent and robust histories. This has some resemblence to the consistent histories approach to standard quantum theory by Griffiths [12], Omnès [13] and Gell-Mann and Hartle [14].

There is a limit to the usefulness of these complete set insertions. For the action $S^{(n)}$ becomes increasingly flatter, so that fluctuations or deviations from the condensate become more extreme, suggesting that any time-like sequencing description has limited relevance at very short time intervals. This resolves the objections of Parmenides and Zeno to infinite 'information processing'. So in a HQS the modelling of time by the real number line will be limited by the nature of the fluctuation dominance that sets in at too fine a resolution: the very concept of spacetime simply dissolves away into a flux of non-geometric fluctuations. At intermediate scales the HQS would appear to produce a spacetime modelling resembling a foamy or fractal structure.

HQS is necessarily a quantum cosmology. But quantum cosmology is not just about the beginning of the universe; it is about the ongoing evolution of the universe. It is a part of the classicalisation process that classical (continuum) differential equations can be used to evolve 3-spaces etc, but that phenomenology is contingent upon the underlying Heraclitean Quantum System; as Heraclitus suggested: the appearance of 'things' depends upon this flux for their continuity and identity.

We have not discussed quantum 'matter': matter will be excitations embedded in the spatial quantum structures. Space is not passive, it is not a container. The container role of the classical manifolds will finally emerge when we integrate out the HQS fine detail, resulting in the traditional local field theory formulations.

We thank Susan Gunner for her support.

\section{References}

[1] J. A. Wheeler, Pregeometry: Motivations and prospects, in Quantum Theory and Gravitation, A. R. Marlow, ed. (Academic Press, New York 1980). 
[2] P. Gibbs, The small scale structure of space-time: A bibliographical review, hepth/9506171.

[3] C. Isham, Structural issues in quantum gravity, gr-qc/9510063.

[4] R.T. Cahill, Aust. J. Phys. 42(1989)171; Nucl. Phys. A 543(1992)63c.

[5] R. T. Cahill and S. M. Gunner, Phys. Lett. B 359(1995)281; Mod. Phys. Lett. A 39(1995)3051; The global colour model of QCD and its relationship to the NJL model, chiral perturbation theory and other models, hep-ph/9601319; The pion mass formula, hep-ph/9602240.

[6] M. R. Frank and T. Meissner, Low energy QCD: Chiral coefficients and the quarkgluon interaction, nucl-th/9511016.

[7] G. Leibniz, The monadology in Leibniz, Philosophical Writings ed. G. H. R. Parkinson, (Dent, London 1973).

[8] F. A. Berezin The method of second quantisation, (Academic Press, New York 1966).

[9] G. Nagels, Gen. Rel. and Grav. 17(1985)545.

[10] S. W. Hawking, Nucl. Phys. B 144(1978)349; Phys. Rev. D 37(1988)904; Phys. Rev. D 53(1996)3099.

[11] S. Coleman, Nucl. Phys. B 130(1988)643.

[12] R. B. Griffiths, J. Stat. Phys. 36(1984)219.

[13] R. Omnès, Rev. Mod. Phys. 64(1992)339.

[14] M. Gell-Mann and J. Hartle, "Quantum mechanics in the light of quantum cosmology" in Proc. of the 3rd Int. Symp. on the Foundations of Quantum Mechanics in the Light of New Technologies, (Phys. Soc. of Japan, Tokyo, Japan, 1990), pp321-343. 